\title{
Urolithiasis in Germany: Trends from the National DRG Database
}

\author{
Hendrik Heers $^{a}$ David Stay ${ }^{b}$ Thomas Wiesmann ${ }^{b}$ Rainer Hofmann ${ }^{a}$ \\ aDepartment of Urology and Paediatric Urology, Philipps-Universität Marburg, Marburg, Germany; ${ }^{\mathrm{b}}$ Department of \\ Anaesthesiology and Intensive Care Medicine, Philipps-Universität Marburg, Marburg, Germany
}

\section{Keywords}

Urolithiasis · Endourology · Epidemiology · Public health patients with increased risk for complications, this results in a higher monetary burden to the health care system and society.

(C) 2021 The Author(s)

Published by S. Karger AG, Basel

\begin{abstract}
Introduction: Urolithiasis is a common disease leading to a high socioeconomic burden due to treatment costs and sickness leave. The aim of this study was to evaluate recent trends in the incidence of urolithiasis in Germany and in the use of therapeutic interventions. Methods: Treatment data for all in-patient hospital episodes for urolithiasis between 2005 and 2016 were extracted from the national DRG statistics at DESTATIS and analysed with regard to the corresponding procedures according to the OPS code. Results: Incidence for urolithiasis was stable at around 120,000 cases per year during the observation period with a male:female ratio of 2:1. Rising numbers were noted for patients $>80$ years. Nevertheless, the number of coded procedures rose significantly with a marked disproportionate transition from extracorporeal shock wave lithotripsy towards ureterorenoscopy. Percutaneous nephrolithotomy was performed more frequently on a smaller scale. Discussion/Conclusion: While the global incidence of urolithiasis is still rising, Germany, as other Western countries, has reached a plateau. There is a remarkable trend towards invasive treatment of even asymptomatic kidney stones. Besides the effects on individual
\end{abstract}

\section{Introduction}

Urolithiasis is a very common disease. The global incidence has been rising for years, whereas a plateau seems to be reached in Western countries as was reported in studies from the United Kingdom, Iceland, and Taiwan; however, some of these studies report a mixed picture for different parts of the population with differing incidences according to gender and/or race [1-8]. The life time prevalence in Western Europe is around 10\% [7]. The aetiology is complex and involves genetic factors and predisposing conditions as well as dietary and drinking habits and life style factors (overweight and lack of exercise). Thus, efficient metaphylaxis requires excellent compliance, and many patients suffer from recurring stone episodes.

While most kidney stones remain asymptomatic, the majority of patients experiencing stone passage into the ureter will seek care. Spontaneous passage may be expected in small stones up to $5 \mathrm{~mm}$ under symptomatic treat-
C 2021 The Author(s).

Published by S. Karger AG, Basel

This is an Open Access article licensed under the Creative Commons Attribution-NonCommercial-4.0 International License (CC BY-NC) (http://www.karger.com/Services/OpenAccessLicense), applicable to the online version of the article only. Usage and distribution for commercial purposes requires written permission.
Correspondence to:

Hendrik Heers, heers@med.uni-marburg.de 
ment [9]. Interventional treatment is required for larger stones, refractory pain, or complications (infected obstructed kidney and acute kidney injury).

The technical advances in endourology over the past 40 years have led to dramatic changes in the treatment of urolithiasis. The introduction of extracorporeal shock wave lithotripsy (SWL) in 1980 by the Munich group around Christian Chaussy was a revolution and for the first time allowed non-invasive treatment also of asymptomatic kidney stones before they could induce a renal colic [10]. Further developments led to the introduction of ureterorenoscopy (URS) which made stones in all positions of the collecting system accessible to endoscopic surgery. Lithotripsy techniques became less traumatic with Ho:YAG laser being the current gold standard [9]. Percutaneous nephrolithotomy (PCNL) was developed for the treatment of large kidney stones and has recently experienced a miniaturization of access tract and instruments.

While the treatment of urolithiasis is subject to constant innovation, there are little new data with regard to pathogenesis and prevention. Especially the literature on dietary recommendations is limited and contradictory. Best established are recommendations on increased fluid intake resulting in a daily urine output of at least $2.5 \mathrm{~L}$ [11].

Although the modern treatment options are less invasive and more comfortable for both patients and care team, they result in substantial costs to the health care systems. On top of that, the socioeconomic burden caused by sickness leave is considerable.

In order to enable demand-oriented planning and resource allocation within the health care sector, it is helpful to observe real-world data on the clinical treatment and to mark changes and trends in the therapy of common diseases. While data have been published for several comparable countries, there is no recent analysis of urolithiasis treatment in Germany to our knowledge $[7,8,12]$. The DRG statistics from the federal statistics bureau (DESTATIS) collects diagnosis and procedural codes for all inpatient hospital cases in Germany according to the ICD10 and OPS coding systems. Thus, it enables insight into changes of the incidence of diseases as well as changes in the choice of therapeutic interventions. Using this methodology, conditions from different specialities including neurology and intensive care have recently been analysed $[13,14]$. The aim of this study was to investigate changes in the incidence and treatment of urolithiasis in Germany for the period 2005-2016.

\section{Materials and Methods}

Data from the DRG statistics at DESTATIS were extracted for the time period 2005-2016. The ICD-10 codes relevant for urolithiasis were selected when used as main diagnosis: N20.0 (kidney stone), N20.1 (ureteric stone), N20.2 (kidney stone with ureteric stone), N20.9 (urinary calculus, unspecified), and N23 (unspecified renal colic). All procedures linked to the individual cases were collected and classified according to the OPS classification of the German institute of medical documentation and information (DIMDI) in the version of the corresponding year and filtered for the procedures relevant to the diagnosis. This included URS (OPS codes 5-562.4, 5-562.5, 5-562.8, 5-550.21, and 5-550.31), PCNL (5$562.6,5-562.7,5-550.20$, and 5-550.30), and SWL (8-110.1 and $8-110.2)$ as well as the auxiliary measures insertion of ureteric stent (8-137.0) and percutaneous nephrostomy (5-550.1 and 5-550.6). Open stone surgery of the upper urinary tract (5-562.0, 5-562.1, 5-550.0, 5-550.2, 5-550.4, and 5-550.5) and the historic procedure of loop extraction of ureteric stones were also included (5-562.2 and 5-562.3). Other available data that were collected concerned the age cohort, sex, type of discharge, and federal state where the treatment was carried out.

A descriptive retrospective population-based analysis of the extracted anonymized data of in-patient hospital episodes was performed using Excel (version 16; Microsoft Corporation, Redmond, WA, USA) and GraphPad Prism (version 7; GraphPad Software Inc., La Jolla, CA, USA). The manuscript was drafted in accordance with the RECORD statement [15].

\section{Results}

Similar to reports from other countries, Germany has shown a stable incidence for urolithiasis in recent years (Fig. 1). The main diagnosis N20.0 (kidney stone) was coded in 41,746 cases in 2005 and in 45,370 cases in 2016, whereas the numbers for $\mathrm{N} 20.1$ (ureteric stone) were minimally declining from 64,563 to 62,066 during the same time. A distinct 3 -fold rise from 2,814 to 8,528 could be noted for the diagnosis N20.2 (kidney stone with ureteric stone). The overall incidence was stable for both male and female patients and in all age cohorts (Fig. 2). Only a constant rise by about $50 \%$ in those above 80 years was observed, from 3,273 to 4,680 cases. Given an estimated population of 83.1 million in Germany as currently stated by DESTATIS, the overall annual incidence for urolithiasis was relatively stable at $0.147 \%$ in 2005 and $0.153 \%$ in 2016 or 1,474 cases per 1 million inhabitants in 2005 and 1,527 in 2016.

The average length of stay declined from 3.7 days in 2005 to 2.6 days in 2016. Most recently, a plateau seems to have been reached (Fig. 3). The shortening of the length of stay was most obvious for the main diagnosis N20.2 (kidney stone with ureteric stone). The most extensive 


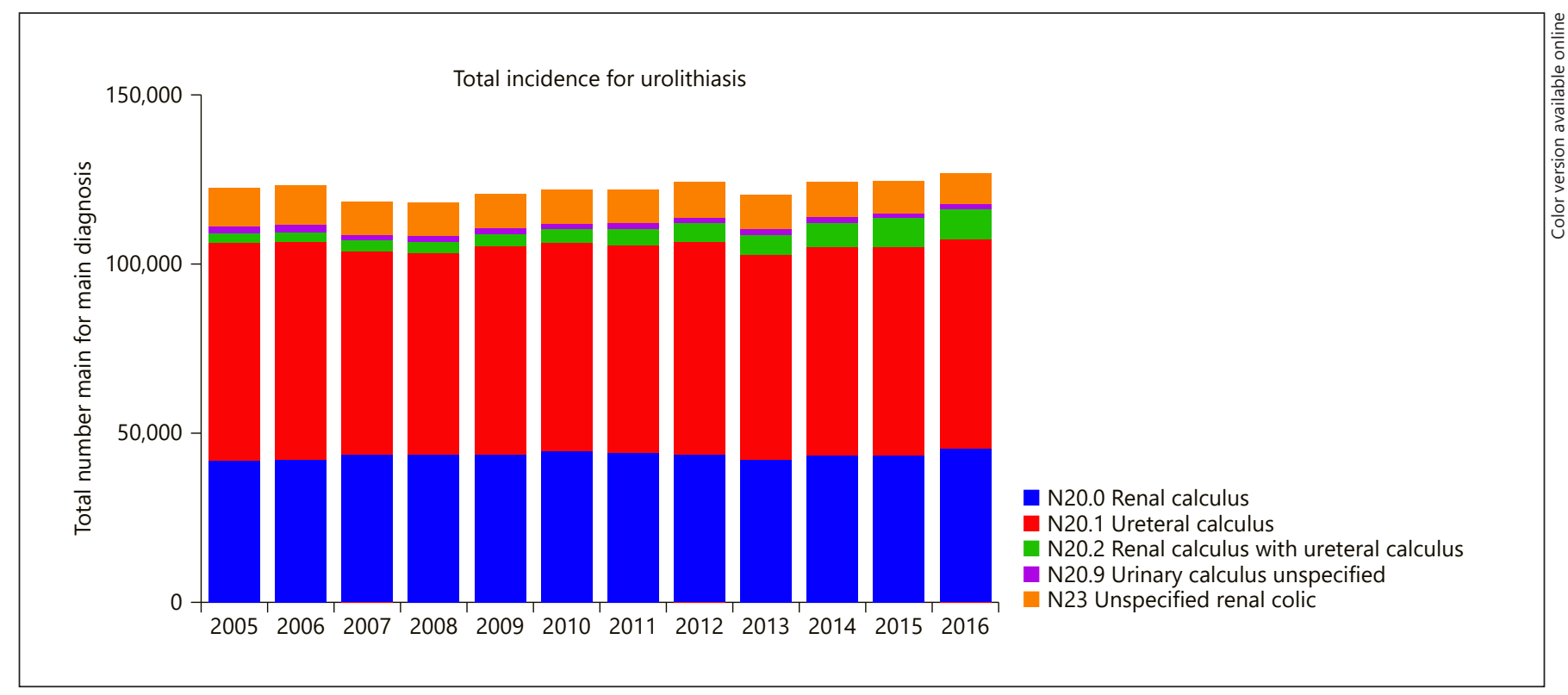

Fig. 1. Overall incidence for urolithiasis in each year as marked by main diagnosis.

Fig. 2. Gender-specific overall incidence

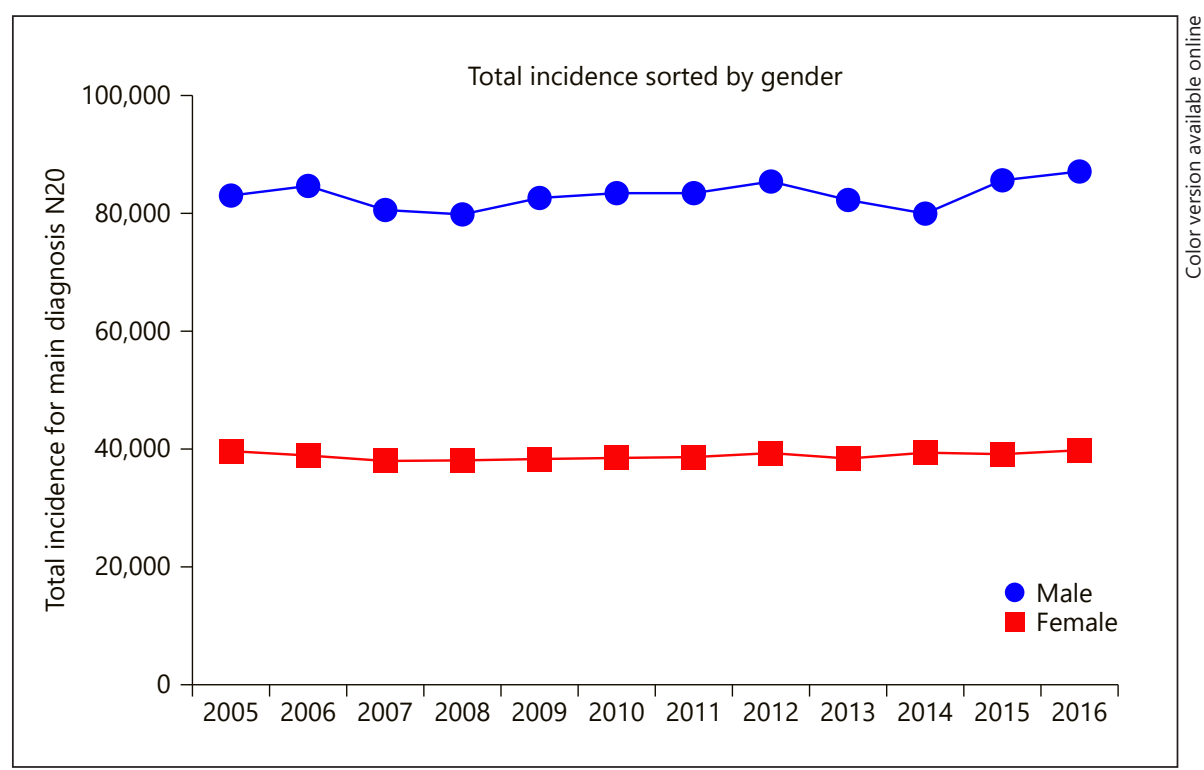
for urolithiasis.

length of stay could be observed in the age cohort $>80$ years.

Given an overall comparatively stable incidence, there was a marked rise in the number of coded procedures for all relevant main diagnoses. Figure 4 shows the total number of OPS-coded procedures for kidney stones (N20.0) and ureteric stones (N20.1). While the amount of interventions for ureteric stones rose by $63.6 \%$ from 118,906 in 2005 to 194,486 in 2016, the increase for kidney stones was even more distinct with $270 \%$ from 68,666 to 254,023 . The clearest increment could be seen in procedures for coincident kidney and ureteric stones (N20.2). Numbers rose from 5,624 to 39,796 by $>600 \%$.

Figure 5 shows the trends for individual techniques in stone treatment. A long-term trend away from SWL towards endoscopic stone treatment can be observed. The 
Fig. 3. Average length of hospital stay for all mentioned main diagnoses over time.
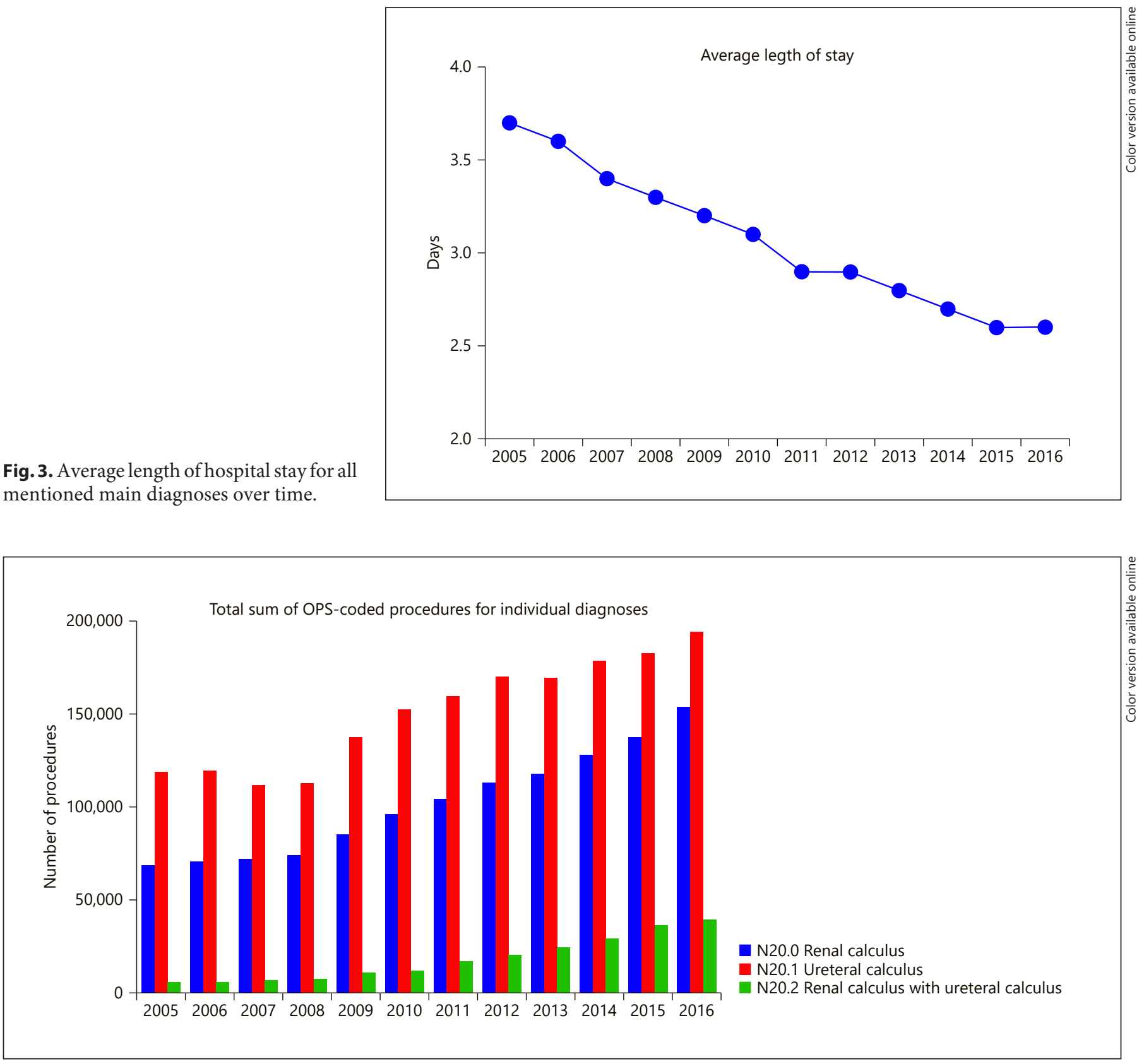

Fig. 4. Total numbers of OPS-coded procedures for individual diagnoses over time.

number of SWL treatments for kidney stones dropped by more than half from 24,305 to 10,162 . This was even clearer in SWL for ureteric stones (from 6,578 to 1,552). URS was employed for ureteric stones in 20,000 cases in 2005 ; in 2016, there were 28,742 cases. The strongest increase was seen in URS for kidney stones. The numbers rose from 2,645 to 18,516 by almost $600 \%$. PCNL as a technique for the treatment of large kidney stones was only registered from 2008 onward. During the time until 2016 , the annual cases almost doubled from 3,711 to 6,798 . Only very small numbers were noted for open-surgical stone removal and loop extraction (1,811-855 and 1,209-540, respectively).

In terms of auxiliary measures, ureteric stent insertion and nephrostomy insertion were also analysed. Ureteric stenting is much more common. Although numbers in 
Fig. 5. Temporal trends for individual

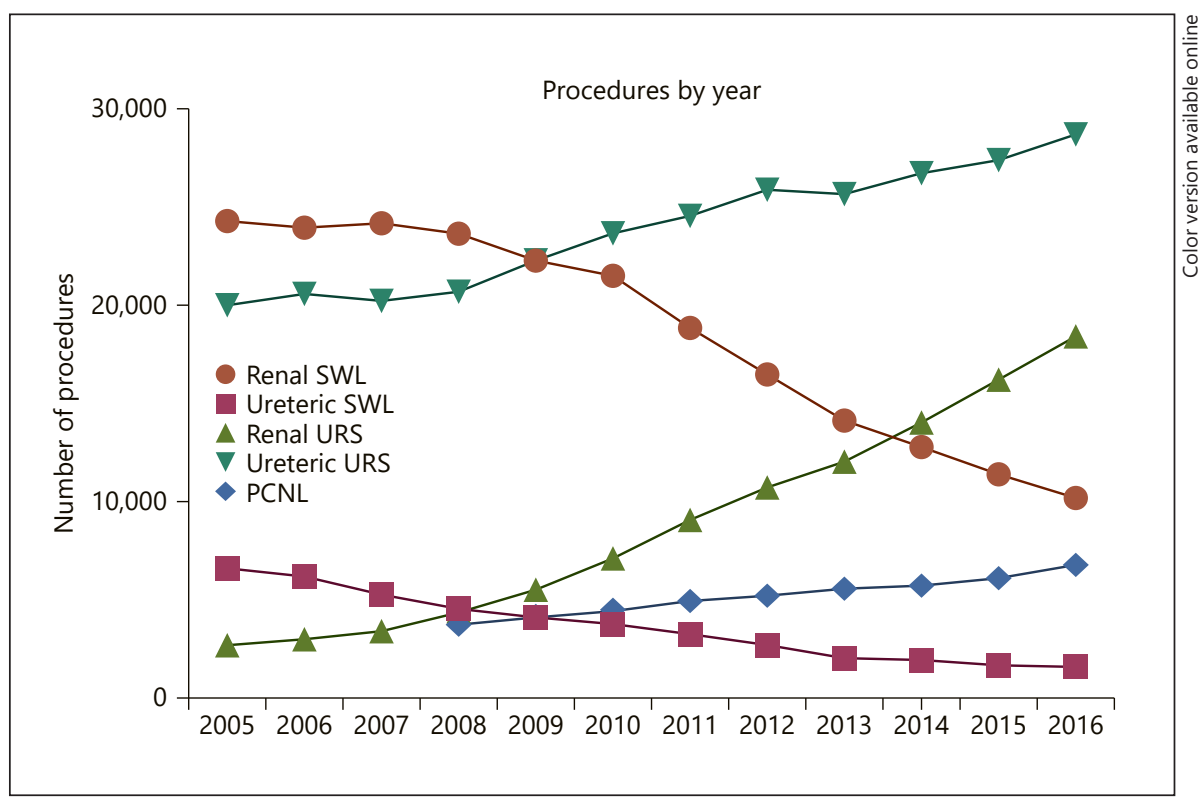
OPS-coded procedures in stone treatment.

patients with ureteric stones were more or less stable (from 19,873 to 23,025 ), stenting is used more frequently for kidney stones $(5,411$ to 12,039$)$ and for coincident kidney and ureteric stones ( 839 to 3,656 ). The amount of nephrostomy insertions is much smaller and also showed an increase from 1,894 to 4,098 cases.

Ninety-three percent of all stone patients experienced a regular hospital discharge. This was constant during the observation period. A transfer to a different hospital occurred in $4.2 \%$ of cases. The fraction of patients leaving the hospital against medical advice doubled from 1.11 to $2.25 \%$. Deaths during in-patient treatment were rare. However, an increase from 6 cases in 2005 to 41 cases in 2016 was noted.

\section{Discussion/Conclusion}

Data from the DRG statistics are no perfect representation of the clinical normality in Germany. Only in-patient cases are registered, and accurate coding of diagnoses and procedures by the care team is required. A range of factors including monetary incentives might influence coding practice. However, these flaws are similar to those of other national hospital episode statistics, after all. It can be assumed that errors in data entry are comparable over time, and hence conclusions on trends in the incidence and use of different procedures can be drawn as has already been shown for other diseases $[13,14]$.
The overall incidence for urolithiasis in Germany was stable with the only considerable rise in the age cohort $>80$ years. This is consistent with the rising life expectation. A further increase of cases in this age cohort can be expected. The more common active treatment of geriatric multimorbid patients may be an explanation for the rising number of deaths during hospital stays for urolithiasis as they are more prone to complications from treatment such as sepsis or bleeding. This needs to be taken into account when interventional stone treatment is planned, and the least invasive treatment modality should be chosen if feasible. During endoscopic surgery, lithotripsy time should be limited. It is sometimes safer to remove the stone burden in several sessions rather than on the first attempt. Nevertheless, while the percentage increase in deaths is significant (5.8-fold), the absolute numbers are on a very low level, and lethal complications remain an exception.

A striking increase of the main diagnosis N20.2 (kidney stone with ureteric stone) was observed. This suggests that (asymptomatic) kidney stones are more often actively treated coincidently during procedures for ureteric stones as shown in other countries [7]. Usually, ureteroscopy is extended by flexible endoscopy of the kidney for the treatment of calyceal stones. This results in longer operating time and potentially in a higher risk for complications such as septicaemia and ureteric injury $[16,17]$.

The falling case numbers for N20.9 (urinary calculus, unspecified) and N23 (unspecified renal colic) can possibly be explained by better diagnostic technique due to 
the widespread use of low-dose CT with modern protocols which also allow for conclusions on stone composition [18-20]. Another factor might be better coding quality with growing experience with the DRG system. The shift from plain X-ray and i.v. urogram towards CT KUB is also probably the main reason for the growing detection of asymptomatic kidney stones and thus of their active treatment.

More men than women are afflicted by urolithiasis (in a ratio of 2:1). This has been constant over the past few years, unlike in other countries such as the USA where there is a growing incidence in women [3]. In general, lifestyle and diet are important factors [11,21]. Urolithiasis is a classic lifestyle disease. While the incidence in threshold countries and countries of rapidly growing wealth is rising, many developed countries report stable incidences or, such as Taiwan, even falling numbers [7, 22-24].

The average length of stay shortened significantly during the observation period, but the curve flattened somewhat. The most obvious explanation for that is the minimal length of stay required within the DRG system.

In the choice of treatment for upper tract stone disease, there is a marked shift from SWL towards URS. Similar observations were made in other Western countries $[1,3$, $6,12]$. This trend is driven by both physician-related factors and patient-specific factors, especially the wish for rapid complete stone removal in 1 session and short hospital stays which is also triggered by socioeconomic pressure. There certainly is an influence by the fact that constant technical advancements particularly in URS make this procedure very attractive, whereas SWL has remained more or less unchanged in recent years. In addition to that, aspects of remuneration specific to Germany and its DRG system are less favourable to SWL. This leads to fewer expensive SWL machines being available in German hospitals. Nevertheless, it must be noted that the DRG statistics only report in-patient treatment. While URS is usually carried out in this setting, there may be a certain amount of outpatient SWL treatments which were not recorded. Unfortunately, those numbers are not available. Thus, while the decrease in SWL is certainly real, the scale may possibly be less dramatic than suggested.

The increase in numbers for PCNL is much slower and on a lower level, although its miniaturization enables the treatment of smaller kidney stones while it used to be a technique reserved for large staghorn stones in the past. Open stone surgery today is a rare occurrence and remains an option for select complex cases in high-volume centres.
The increasingly invasive therapy of asymptomatic kidney stones is subject to discussion. The mantra of complete stone removal has been preached within the endourologic community in recent years. While fragments $<2-4 \mathrm{~mm}$ were considered "clinically insignificant" during the high time of SWL, the aim of modern URS surgeons is to leave behind no visible stone fragments at all to lower the risk of stone recurrence. This is furthermore enhanced by the widespread use of low-dose CT for follow-up instead of conventional KUB X-ray. The pros and cons as well as the yet non-uniform definition of "stone free" are being discussed controversially [25-27]. Another aspect is that the rise of endoscopic treatment potentially causes more frequent and prolonged ureteric stenting with corresponding morbidity [28]. This is even more relevant as pre-stenting before URS is very common in Germany, among other reasons due to the DRG system. Up to $80 \%$ of German URS patients undergo pre-stenting [28]. This is not only the case for the sole treatment of kidney stones where primary endoscopic access into the kidney is often unsuccessful but also for the treatment of ureteric stones. Patients are often counselled by their physicians to also have asymptomatic kidney stones removed before they become symptomatic. This choice is probably often fostered by a lack of information and insufficient medical guidance due to the abovementioned mantra.

URS and SWL appear to be equally efficient in the treatment of small- and medium-sized kidney stones [29]. Before reaching a decision on the active treatment of asymptomatic kidney stones, patients should be counselled in depth on all therapeutic options taking into account the natural course. It needs to be noted that about $30 \%$ of patients with asymptomatic nephrolithiasis develop symptoms over time [30]. Thus, active surveillance is a valid alternative. This aspect is particularly noteworthy during a worldwide pandemic where medical resources need to be preserved and waiting times for elective procedures are increasing.

\section{Statement of Ethics}

The authors confirm that this study was conducted in accordance with the World Medical Association Declaration of Helsinki. This retrospective review of patient data did not require ethical approval by the Local Clinical Ethics Committee of Marburg University Hospital in accordance with local/national guidelines. Written informed consent from participants was not required in accordance with local/national guidelines by the Local Clinical Ethics Committee of Marburg University Hospital. Only fully anonymized aggregated patient data were used. 


\section{Conflict of Interest Statement}

H. Heers discloses honoraria for talks and consultancies by Pfizer, Medac, and Ipsen. T. Wiesmann discloses consultancies for Ratiopharm, Vygon, and Pajunk. The other authors declare no conflicts of interest.

\section{Funding Sources}

No separate funding was obtained for this study.

\section{Author Contributions}

The study was conceived of by H. Heers and T. Wiesmann. Data collection and analysis was performed by D. Stay, H. Heers, T. Wiesmann, and R. Hofmann. The manuscript was drafted by $\mathrm{H}$. Heers and T. Wiesmann and was critically revised by D. Stay and R. Hofmann.

\section{Data Availability Statement}

All data analysed during this study are included in this article. Any additional raw data extracted from DESTATIS remain with the authors and can be accessed upon request by contacting the corresponding author.

\section{References}

1 Prezioso D, Illiano E, Piccinocchi G, Cricelli C, Piccinocchi R, Saita A, et al. Urolithiasis in Italy: an epidemiological study. Arch Ital Urol Androl. 2014;86(2):99-102.

2 Bauer J, Kahlmeyer A, Stredele R, Volkmer BG. Inpatient therapy of urinary stones in Germany: development of the G-DRG system. Urologe A. 2014;53(12):1764-71.

3 Ghani KR, Sammon JD, Karakiewicz PI, Sun M, Bhojani N, Sukumar S, et al. Trends in surgery for upper urinary tract calculi in the USA using the nationwide inpatient sample: 19992009. BJU Int. 2013;112(2):224-30.

4 Scales CD Jr, Smith AC, Hanley JM, Saigal CS; Urologic Diseases in America Pathalogy. Prevalence of kidney stones in the United States. Eur Urol. 2012;62(1):160-5.

5 Seklehner S, Laudano MA, Jamzadeh A, Del Pizzo JJ, Chughtai B, Lee RK. Trends and inequalities in the surgical management of ureteric calculi in the USA. BJU Int. 2014;113(3): 476-83.

6 Seklehner S, Laudano MA, Del Pizzo J, Chughtai B, Lee RK. Renal calculi: trends in the utilization of shockwave lithotripsy and ureteroscopy. Can J Urol. 2015;22(1):762734.

7 Heers H, Turney BW. Trends in urological stone disease: a 5-year update of hospital episode statistics. BJU Int. 2016;118(5):785-9.

8 Chewcharat A, Curhan G. Trends in the prevalence of kidney stones in the United States from 2007 to 2016: Urolithiasis; 2020.

9 Türk CN A, Petrik A, Seitz C, Skolarikos A, Thomas K. EAU Guidelines on Urolithiasis (limited text update March 2020). Amsterdam: EAU Annual Congress; 2020.

10 Chaussy C, Brendel W, Schmiedt E. Extracorporeally induced destruction of kidney stones by shock waves. Lancet. 1980;2(8207):12658.

11 Littlejohns TJ, Neal NL, Bradbury KE, Heers $\mathrm{H}$, Allen NE, Turney BW. Fluid intake and dietary factors and the risk of incident kidney stones in UK biobank: a population-based prospective cohort study. Eur Urol Focus. 2019.

12 Isotani S, Noma Y, Wakumoto Y, Muto S, Horie S. Endurological treatment trend of upper urinary urolithiasis in Japan from the Japanese diagnosis procedure combination database. Int J Urol. 2019;26(10):1007-8.

13 Nimptsch U, Mansky T. Trends in acute inpatient stroke care in Germany: an observational study using administrative hospital data from 2005-2010. Dtsch Arztebl Int. 2012; 109(51-52):885-92.

14 Wiesmann T, Hoenl D, Wulf H, Irqsusi M. Extracorporeal liver support: trending epidemiology and mortality: a nationwide database analysis 2007-2015. BMC Gastroenterol. 2019;19(1):160

15 Benchimol EI, Smeeth L, Guttmann A, Harron K, Hemkens LG, Moher D, et al. The reporting of studies conducted using observational routinely-collected health data (RECORD) statement. Z Evid Fortbild Qual Gesundhwes. 2016;115:33-48.

16 Ulvik O, Harneshaug JR, Gjengsto P. Ureteral strictures following ureteroscopic stone treatment. J Endourol. 2020.

17 Chugh S, Pietropaolo A, Montanari E, Sarica K, Somani BK. Predictors of urinary infections and urosepsis after ureteroscopy for stone disease: a systematic review from eau section of urolithiasis (EULIS). Curr Urol Rep. 2020;21(4):16.

18 Nestler T, Haneder S, Grosse Hokamp N. Modern imaging techniques in urinary stone disease. Curr Opin Urol. 2019;29(2):81-8

19 Cui HW, Tan TK, Christiansen FE, Osther PJS, Turney BW. The utility of automated volume analysis of renal stones before and after shockwave lithotripsy treatment. Urolithiasis. 2020.

20 Nourian A, Ghiraldi E, Friedlander JI. Dualenergy ct for urinary stone evaluation. Curr Urol Rep. 2020;22(1):1.
21 De SK, Liu X, Monga M. Changing trends in the American diet and the rising prevalence of kidney stones. Urology. 2014;84(5):1030-3.

22 Robertson WG. Stone formation in the Middle eastern gulf states: a review. Arab J Urol. 2012;10(3):265-72.

23 Edvardsson VO, Indridason OS, Haraldsson G, Kjartansson O, Palsson R. Temporal trends in the incidence of kidney stone disease. Kidney Int. 2013;83(1):146-52.

24 Huang WY, Chen YF, Carter S, Chang HC Lan CF, Huang KH. Epidemiology of upper urinary tract stone disease in a Taiwanese population: a nationwide, population based study. J Urol. 2013;189(6):2158-63.

25 Wilhelm K, Hahn O, Schoenthaler M, Hein S, Neubauer J, Schnabel M, et al. Stone-free rate after treating kidney stones exceeding $10 \mathrm{~mm}$ via flexible ureteroscopy: can endoscopic assessment replace low-dose computed tomography control? Urol Int. 2019;103(3):326-30.

26 York NE, Zheng M, Elmansy HM, Rivera ME Krambeck AE, Lingeman JE. Stone-free Outcomes of flexible ureteroscopy for renal calculi utilizing computed tomography imaging. Urology. 2019;124:52-6.

27 Ulvik O, Harneshaug JR, Gjengsto P. What do we mean by "stone free," and how accurate are urologists in predicting stone-free status following ureteroscopy? J Endourol. 2021.

28 Reicherz A, Sahin R, Häuser L, Noldus J, Bach P. An empirical study on the operative treatment of symptomatic urolithiasis in Germany. Urol Int. 2021;105(3-4):1-7.

29 Srisubat A, Potisat S, Lojanapiwat B, Setthawong V, Laopaiboon M. Extracorporeal shock wave lithotripsy (ESWL) versus percutaneous nephrolithotomy (PCNL) or retrograde intrarenal surgery (RIRS) for kidney stones. Cochrane Database Syst Rev. 2014;11: CD007044

30 Streeper NM. Asymptomatic renal stones-to treat or not to treat. Curr Urol Rep. 2018; 19(5):29. 